\title{
Attention in Skilled Behavior: an Argument for Pluralism
}

\author{
Alex Dayer ${ }^{1} \cdot$ Carolyn Dicey Jennings $^{2}$
}

Accepted: 2 February 2021 /Published online: 16 March 2021

(C) The Author(s) 2021, corrected publication 2021

\begin{abstract}
Peak human performance - whether of Olympic athletes, Nobel prize winners, or you cooking the best dish you've ever made - depends on skill. Skill is at the heart of what it means to excel. Yet, the fixity of skilled behavior can sometimes make it seem a lower-level activity, more akin to the movements of an invertebrate or a machine. Peak performance in elite athletes is often described, for example, as "automatic" by those athletes: "The most frequent response from participants (eight athletes and one coach) when describing the execution of a peak performance was the automatic execution of performance" (Anderson et al. 2014). While the automaticity of skilled behavior is widely acknowledged, some worry that too much automaticity in skill would challenge its ability to exhibit human excellence. And so two camps have developed: those who focus on the automaticity of skilled behavior, the "habitualists," and those who focus on the higher-level cognition behind peak performance, the "intellectualists." We take a different tack. We argue that skilled behavior weaves together automaticity and higherlevel cognition, which we call "pluralism." That is, we argue that automaticity and higher-level cognition are both normal features of skilled behavior that benefit skilled behavior. This view is hinted at in other quotes about automaticity in skill—while expert gamers describe themselves as "playing with" automaticity (Taylor and Elam 2018), expert musicians are said to balance automaticity with creativity through performance cues: "Performance cues allow the musician to attend to some aspects of the performance while allowing others to be executed automatically" (Chaffin and Logan 2006). We describe in this paper three ways that higher-level cognition and automaticity are woven together. The first two, level pluralism and synchronic
\end{abstract}

Alex Dayer and Carolyn Dicey Jennings contributed equally to this work.

Authors listed alphabetically

Carolyn Dicey Jennings

Cjennings3@ucmerced.edu

1 Cognitive and Information Sciences, University of California, Merced, 5200 N. Lake Road, Merced, CA 95343, USA

2 University of California, 5200 N. Lake Road, Merced, CA 95343, USA 
pluralism, are described in other papers, albeit under different cover. We take our contribution to be both distinguishing the three forms and contributing the third, diachronic pluralism. In fact, we find that diachronic pluralism presents the strongest case against habitualism and intellectualism, especially when considered through the example of strategic automaticity. In each case of pluralism, we use research on the presence or absence of attention (e.g., in mind wandering) to explore the presence or absence of higher-level cognition in skilled behavior.

\section{Introduction}

Let's consider the example of knitting a hat for a friend, which we will refer to throughout the paper. Whereas a novice knitter pays effortful attention to the specific motions required to form a single stitch, the practiced knitter does not need to do so, and can even carry on a conversation or watch a show without disrupting the now relatively effortless, automatic process. ${ }^{1}$ Unlike the novice knitter, the practiced knitter may fail to remember the specifics of a knitting session, in part due to engagement in these other activities. On the other hand, the practiced knitter may engage attention in the broader knitting project, particularly if it has difficult stitch or yarn changes. The practiced knitter may use cues to help guide those changes, such as strategic advice the knitter has learned from others, but the practiced knitter may also use elements from a mind wandering episode. The practiced knitter may, for example, think about how much their friend likes stripes during a mind wandering episode and thus decide to knit and purl alternating rows. This can be seamlessly integrated during the knitting process - the knitter might make the change to a different stitch for a new row with minimal disruption, without an onlooker noticing the change. Yet, the change will have improved the hat's appeal to the friend. Thus, in contrast to attention, while mind wandering in the novice knitter may be disruptive, mind wandering in the practiced knitter can improve the overall outcome. As this example demonstrates, attention, mind wandering, higher-level cognition, and automaticity are all a normal part of skilled behavior, which we will describe in more detail below.

Before proceeding, we find it necessary to provide some conceptual background on the terms used throughout this paper. First, "intellectualism" is used here somewhat differently than in some other papers. Namely, there is a debate regarding the division of know-how from know-that, with "intellectualism" describing the position that all forms of know-how are forms of know-that (this debate is discussed in, e.g., Levy 2017). In parallel with this debate, involving some of the same philosophers, is one on whether skill necessarily involves higher-level cognition - a second type of "intellectualism." It is the latter debate and the latter form of intellectualism that we are discussing here.

\footnotetext{
${ }^{1}$ Here, and throughout the paper, we use the standard definition of automaticity: "The ability to perform a task by automatic processing, independent of conscious control and attention. Strong automaticity is almost entirely automatic and can be carried out without attention. Partial automaticity refers to processing that can be performed without conscious control, but which is performed better with attention." (Automaticity, 2020; emphasis ours). This is the definition typically used by psychologists (e.g. Schneider and Shiffrin 1977).

2 That this is a separate debate is sometimes explicitly acknowledged by those in the first debate, as in Stanley and Krakauer (2013): "Although we have not addressed the topic in depth here, another position taken by Dreyfus is that true expertise is somehow automatic or habitual, and knowledge is no longer required."
} 
Drawing the distinction between these two debates is important because the form of higher-level cognition that interests us is not coextensive with the propositional cognition of "know-that." "We use the term to capture a broader set of mental capacities, including top-down attention and executive control, which are also invoked in this debate. That is, by "higher-level cognition," we include the propositional structures responsible for thought, reflection, planning, and intention-formation (which are not necessarily deliberate) as well as other higher-level cognitive processes such as voluntary attention and executive control (Miller and Wallis 2009). One might see ours as the view that higher-level cognition can be about content or control.

Other papers in this debate have set the contrast as between, for example, "higher cognition" and "cognitive processes," on the one hand, and "mindless" and "unreflective" activity with "little conscious awareness," on the other (Christensen et al. 2016, 37-8). Such inconsistent wording within and across these papers makes it difficult to tell what is at stake. For clarity, we set our dividing line in this paper between higher-level cognition and automaticity. As described above, higher-level cognition includes both executive control and propositional content, and is used in contrast with automaticity. As for automaticity, we do not assume it to be mindless or unconscious, even while we do assume it is devoid of executive control, at least in its strongest forms (see footnote 2, Jennings 2015). As we mention later in the paper, we agree with, e.g., Fridland (2017) that automaticity is distinguishable from reflex. While we see higher-level cognition and automaticity as highly integrated (as in Christensen et al.'s "systems view"), we also see them as having distinct physical and conceptual features (as in the more classic "dual process view"). ${ }^{4}$ That is, even if it were correct to say that higher-level cognition and automaticity "can be hardly separated," they could still be separated, in our view (Caiani and Ferretti 2017). ${ }^{5}$

In our arguments, we claim that both higher-level cognition and automaticity are a "normal" part of skilled behavior. By "normal" we mean "as a rule." That is, we admit that there may be exceptional cases in which automaticity and higher-level cognition are not both part of skilled behavior, which we do not see as threatening our analysis (see Fig. 1 below). Others sometimes focus on either the issue of presence or absence (of higher-level cognition and automaticity in skilled behavior) or on the issue of improvement or hindrance (of skilled behavior by higher-level cognition and automaticity). In our view, if automaticity and higher-level cognition are both part of skilled behavior as a rule, then their absence from skilled behavior will be a hindrance to that behavior, as a rule. Conversely, if automaticity and higher-level cognition are both beneficial to skilled behavior, we would expect them to be present in skilled behavior, as a rule.

\footnotetext{
${ }^{3}$ This is one reason we don't discuss, for instance, the interface problem, raised by other authors, since that problem concerns the interface between propositional and non-propositional knowledge (see, e.g., Mylopoulos and Pacherie 2017).

${ }^{4}$ Another point of difference with, for example, Christensen et al. (2016) is that we do not take all forms of selectivity and focus to be forms of top-down attention. Take the focus described in cricket batters by Sutton (2007): "The kind of concentration evident in high-quality batting performances, most cricketers think, is not conscious and deliberate attention" (778). We agree with this stance, finding it possible to distinguish the focus and concentration that results from top-down attention from the focus and concentration that results from automatic processing.

${ }^{5}$ But note that Caiani and Ferretti appear to be focused primarily on the purported distinction between the dorsal and ventral streams in vision.
} 


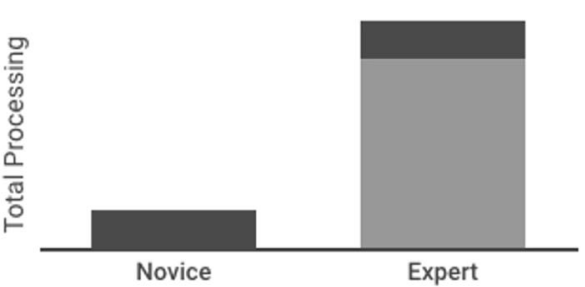

Level Pluralism

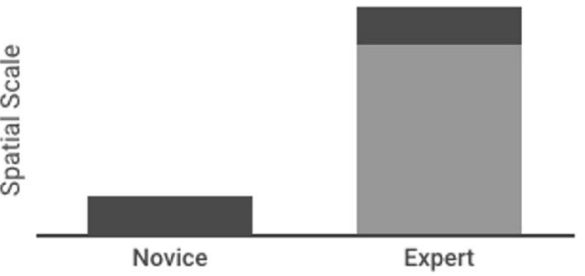

Synchronic Pluralism

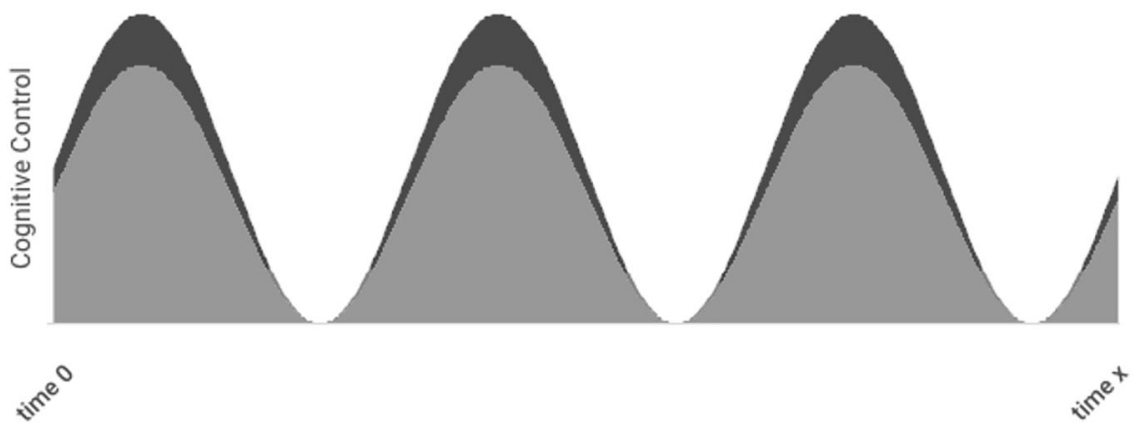

Diachronic Pluralism

Automaticity

Higher-Level Cognition

Fig. 1 A cartoon rendering of the relationship between higher-level cognition and automaticity in task-related, controlled processing for each of the forms of pluralism discussed in this paper. For Level Pluralism, the novice uses primarily higher-level cognition, whereas the expert can also make use of automatized components and thus more total brain processing can be dedicated to the task (see, e.g., Chang 2014). For Synchronic Pluralism the spatial scale that can be covered by higher-level cognition for the novice (e.g. one part of the visual scene at a time) is smaller than that covered by higher-level cognition for the expert (e.g. the entire visual scene), since the expert is able to nest the newly automatized components through vertical layering. For Diachronic Pluralism cognitive control fluctuates with time, allowing for task-related spontaneous cognition when cognitive control is low (horizontal layering; note that automatic processing may continue as higher-level cognition wanes, it just won't be "under" it, in a controlled or nested way). In all three forms of pluralism, both automaticity and higher-level cognition are a normal part of skilled behavior

In making our case, we assume that skilled behavior can be analyzed both vertically and horizontally. That is, skilled behavior takes place over a time span that can be decomposed into smaller chunks (horizontal analysis), but it also admits of different spatial scales (vertical analysis) in which task components are nested within a task. ${ }^{6}$ We call this horizontal and vertical layering, respectively. In our view, horizontal and vertical layering occur within skilled behavior. In contrast to this is the view that higher-level cognition, for example, only occurs in the "gaps between" (horizontal) or "gaps above" (vertical) skilled behavior (Bergamin 2017, 410); We contest this below.

\footnotetext{
${ }^{6}$ One description of spatial and temporal scales is provided here: "At the conceptual level, it is intuitively understandable that forms of hierarchy can be realized through differing scales in space and time. In a photo image, for example, elemental information in a narrow space, such as the edges of an object and the color of pixels, is integrated into complex features of the image in a larger space. In speech sounds, syllable-level information on short time scale is integrated into word-level information over a longer time scale. It is not unrealistic to think that the mechanisms of multiple scales in space and time, which are responsible for generating these hierarchies, might also be at work in the neural systems of animals" (Yamashita and Tani 2008).
} 
Another important distinction we make is between task-related processing and controlled processing. We see processing as task-related depending on its content, whereas we see processing as controlled depending on its mechanism. This is important in our discussion of mind wandering and top-down attention, since understanding the primary difference between these to be one of control, rather than task-relevance, helps to explain how mind wandering is able to contribute to skilled behavior. That is, mind wandering is a form of spontaneous cognition that may or may not be task-related depending on its content, whereas top-down attention is a form of control, effortfully drawing resources to the task at hand. As we discuss below, we do not take as evidence for pluralism forms of higher-level cognition that are irrelevant to the skilled task; the higher-level cognition we consider is always connected to the task via either control or content.

As mentioned above, we are not the first to argue for pluralism. In fact, even Stanley and Krakauer (2013) appear to support a form of pluralism: "the musician or athlete is using knowledge of the musical score or the game to dictate to those automatic nonknowledge based components; it is the combination that leads to the skilled performance." Yet, our contribution is to specify some ways in which higher-level cognition and automaticity interact and to introduce diachronic pluralism. Rather than proving that both sides are right, we find this form of pluralism to challenge some key players in the debate so far (e.g. Dreyfus and Montero), and hope to convince others that pluralism is a more promising option worthy of future research.

\section{The Debate: Intellectualism Vs. Habitualism}

Before arguing for a pluralist approach, here we survey arguments for and against the two major theoretical positions on skilled behavior. Papineau (2015), for example, identifies two theoretical camps concerning the relationship between higher-level cognition and skilled behavior: habitualism and intellectualism. Whereas habitualist accounts of skilled behavior take a complete lack of higher-level cognition to be a defining feature (Dreyfus 2007), intellectualist accounts emphasize that experts are frequently reflecting on and deliberately thinking about what they are doing while acting skillfully (Montero 2010). Although Papineau's primary focus is the sports psychology literature, we assume his distinction applies to other domains of skilled behavior as well. In the context of sports, Papineau says habitualist accounts claim that "thought is the enemy of sporting excellence" and "cognitive effort only interferes with skill," whereas intellectualist accounts claim that "sporting performance depends crucially on mental control" (295). Notice that under Papineau's description habitualism eschews "thought" and "cognitive effort" while intellectualism endorses the presence of "mental control." As mentioned above, we use the term "higher-level cognition" instead of these other terms, which can include both propositional knowledge and mental control.

\subsection{Habitualism}

Standard models of skill acquisition suggest that as one acquires a skill, one's actions become more automatic and rely less on rules and heuristics (Anderson 1982; Dreyfus and Dreyfus 1980). In one of the most widely accepted models of behavior, all behavior is at first 
driven by the association between an action and its expected outcome, but later driven by a direct association between stimulus and response: "whereas an action...begins under goaldirected control, the net influence of the action-outcome association declines as the strength of the [stimulus-response] association increases until the influence of the latter exceeds the former and so takes over motor control" (Balleine and Dezfouli 2019). The direct link between stimulus and response allows the behavior to become automatic, or to occur without the benefit of attention and conscious control. Christensen et al. (2016) remark that "influential characterizations of skill acquisition in both psychology and philosophy depict it as a progression from an initial cognitive phase to an expert phase in which performance is largely automatic" (37). In the same paper, Christensen et al. draw on the experience of expert cricketer Kumar Sangakkara; he says that in cricket one must be "mindless" and let automaticity take over "without letting your mind take control" (37).

Dreyfus (2007) is perhaps the most well-known defender of the habitualist account of skilled behavior. His position on skilled behavior is most clearly presented in what is known as the McDowell-Dreyfus debate. Dreyfus (2007) accuses McDowell of succumbing to "the myth of the mental" or the idea that conceptual mindedness permeates our lives, and he argues that "know-how" (knowledge about how to act in the world) is the foundation from which "know-that" (knowledge about matters of fact about the world) can arise. ${ }^{7}$ He points to cases in which experts experience absorbed bodily coping, or what has otherwise been referred to as "flow" (Csikszentmihalyi 1997). During absorbed bodily coping or flow, Dreyfus claims that experts have "a kind of non-mental content that is non-conceptual, nonpropositional, non-rational, and non-linguistic" (352). Because know-that can often interfere with know-how, Dreyfus claims that experts perform best in the absence of higher-level cognition (whether this be in the form of propositional knowledge structures or the exertion of mental control). His account is inspired by ideas from dynamical systems theory and Gibsonian ecological psychology: the mark of expertise is the expert's ability to immediately and automatically respond to the solicitations of affordances without the aid of higher-level cognition, such as representations (a target of dynamical systems theory). ${ }^{8}$ Montero (2010) has referred to this as the "principle of automaticity."

A major advantage of Dreyfus' account is that it seems intuitive-focusing on one's actions too intently can certainly be detrimental at times. Although Dreyfus' arguments do not rely on literature from sports psychology, there are studies from this domain on the phenomenon of choking under pressure that support the view. That is, some of the research on choking under pressure supports habitualism and gives us reason to doubt intellectualism about skilled behavior (Beilock and Gray 2007; Beilock and Carr 2001; Beilock et al. 2002). Choking under pressure refers to a decrease in performance when experts experience pressure or stress in response to a given situation. ${ }^{9}$ There are multiple accounts of choking,

\footnotetext{
${ }^{7}$ This is related to debates between intellectualists (see Stanley and Krakauer 2013) and anti-intellectualists (see Fridland 2013), but Dreyfus' emphasis on automaticity makes his account both habitualist and antiintellectualist.

${ }^{8}$ Affordances are, roughly, possibilities for behavior that reflect the fit between an agent's action capabilities and the properties of the environment (Gibson 2014).

9 There are three major accounts of choking: the distraction account, the explicit monitoring account, and the hybrid account. The distraction account suggests the pressure of a situation causes performers to allocate attention away from the present task. The explicit monitoring account is described above. The hybrid account of choking suggests the distraction account and explicit monitoring accounts are not mutually exclusive and that distraction and explicit monitoring can both contribute to choking depending on a range of situational factors at play during skillful performance (DeCaro et al. 2011).
} 
but particularly supportive of Dreyfus is the explicit monitoring hypothesis. The explicit monitoring hypothesis claims that pressure causes experts to allocate attentional resources to the execution of one's actions in such a way that it is detrimental to performance (Beilock and Carr 2001). Thinking about one's actions thus degrades expert performance on this account, supporting Dreyfus' position.

If habitualism is correct, skilled behavior is most successful when higher-level cognition does not interfere with the automatic, procedural processing underlying action execution. However, the fact that higher-level cognition may be detrimental in some circumstances (such as in particular experimental set-ups in sports psychology experiments) does not entail that all instances of higher-level cognition during skilled behavior are detrimental. That is, even if we accept the findings above, they may apply only in specific circumstances such that there may be other contexts in which skilled behavior involves non-automatic, deliberate cognitive processing.

\subsection{Intellectualism}

Intellectualism is the idea that skilled behavior depends crucially on higher-level cognition and mental control. Montero is perhaps the most avid defender of the intellectualist account of skilled behavior with respect to mental control, and she frequently draws on her past experiences as a ballet dancer in order to argue against habitualism and the principle of automaticity. In Montero (2010), she claims that higher-level cognition is often essential to skilled behavior, and she refers to the idea that thinking about one's bodily movements always disrupts performance as "the Maxim." ${ }^{\prime 0}$ She argues against the Maxim by appealing to inconsistencies in firstperson reports (some experts claim that thinking helps them, while others claim that thinking hurts them), skepticism about the applicability of the findings from sports psychology to other domains of expertise (deliberate thinking may not be detrimental in all forms of expert action), and experts' desire to constantly improve. We find her argument from experts' desire to constantly improve to be the strongest argument against habitualism because it directly confronts the habitualist's claim that automaticity is the defining feature of expertise.

Montero (2010) argues against Dreyfus' Maxim by appealing to kaizen: a Japanese word for experts' desire to constantly improve. She argues that kaizen is what is responsible for experts' tendency to push the boundaries of what is possible in given domains of expertise and that one's ability to actualize the desire to improve relies on actively thinking about what one is doing. That is, automaticity alone cannot explain how experts are able to perform at peak levels, and so we must appeal to the higherlevel cognitive processes responsible for mental control. She uses Tiger Woods as an example: Despite already being the best golfer in the world, he altered his swinging style in order to improve his performance. Although this change of swinging style temporarily degraded performance, he was eventually able to master the new action, and his golfing was substantially improved overall. The habitualist might point to the temporary degradation in performance as evidence against intellectualism; however, Montero insists that the fact that experts can and frequently do focus on the specifics of their actions in order to improve themselves is strong evidence against the Maxim and

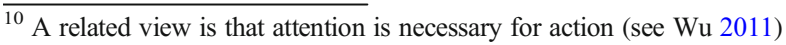


the principle of automaticity. Without higher-level cognition, experts would struggle to discover new, creative ways of acting skillfully that ultimately allow them to reach peak performance levels.

So, it seems that under some circumstances thinking about what you are doing can disrupt expert action (see the sports psychology literature on choking under pressure). However, in other circumstances experts may need to deliberately think about what they are doing in order to adapt to the demands of a given situation or push the boundaries of their domain of expertise. When things are going smoothly or one is content with their level of expertise, the habitualists may be correct about the relationship between higher-level cognition and expert action-higher-level cognition may only serve to interrupt one's actions. However, there are situations in which experts must rely on more than automaticity, and we think intellectualists are correct to emphasize this point.

\section{Pluralism}

Hopefully at this point we have provided a sense of why one might adopt either a habitualist or intellectualist stance on expert action. The habitualists are correct to emphasize that automaticity is a key part of skilled behavior; however, they emphasize this point at the expense of explaining the role of higher-level cognition. The intellectualists are correct to emphasize that higher-level cognition can occur during (and be beneficial to) skilled behavior; however, they do not explain those instances of skilled behavior that proceed without higher-level cognition. Yet, in describing levels of skilled behavior, Dreyfus allows that parts of skilled behavior will be automatized while other parts are not (at least up to the point of mastery), and in rejecting Dreyfus' stronger claims Montero reports that she nonetheless agrees with him that skilled behavior can include automatic components. In fact, the idea that parts, levels, or aspects of skilled behavior are automatic, while other parts, levels, or aspects are not, is commonly found in work on the topic. For the above reasons, we believe neither habitualism nor intellectualism are adequate accounts of skilled behavior. It is our view that a pluralist account of skilled behavior that specifies the relationship between automaticity and higher-level cognition is preferable to existing accounts that focus on either automaticity or higher-level cognition in isolation.

Although not explicitly labeled as such, others have argued for pluralist accounts of skilled behavior. For example, Krakauer (2019) argues that skilled behavior (at any level of expertise) is a combination of "intelligent reflexes" and deliberative decisionmaking processes. Elsewhere, engaging in the debate between intellectualists and antiintellectualists, Levy (2017) has argued for the "composition view" of skilled behavior: he argues that knowledge-how is "composed of a propositional element plus a motor element" (528). Similarly, Buskell (2015) has argued for an account of skill that allows for "the automatic and pre-reflective use of discursive, propositional cues in skill deployment" (1445). Christensen et al. (2016) also defend a hybrid account of skilled behavior in which "cognitive control reduces during skill learning as automatic control comes to play an increasing role, but cognitive control continues to make a substantial positive contribution at advanced levels of skill" (41). Additionally, there is work in phenomenology that resembles both the existing pluralist accounts mentioned above 
and the account we offer here. Høffding (2014) criticized what he refers to as "Dreyfus' dualism," in which higher-level cognition and "skilled coping" are supposed to be incompatible. The crux of Høffding's argument is that there is likely no single phenomenology associated with skilled behavior, rather, it is heterophenomenological. That is, skilled behavior includes moments of automaticity as well as higher-level cognition, such as planning. Whereas Høffding's work focuses on rejecting Drefyus' dualism and creating a taxonomy of possible phenomenological states associated with skilled behavior, the present paper will explicitly argue for a pluralist account of skilled behavior.

The remainder of the paper will be spent articulating different ways one might be a pluralist with respect to skilled behavior, some of which overlap with the accounts mentioned above. We will explore three different conceptions of pluralism concerning skilled behavior: level pluralism, synchronic pluralism, and diachronic pluralism.

\section{Level Pluralism}

Level pluralism is the view that different levels of skill correspond with different degrees of cognitive processing. As was noted at the beginning of Section 2, the standard view is that as one learns a skill, one's actions become increasingly automatic and rely less on higher-level cognition. This is consistent with both Dreyfus' model of skill acquisition and Anderson's ACT-R model (Anderson 1982; Dreyfus and Dreyfus 1980). The standard view is typically considered most compatible with habitualism: whereas novices must explicitly think about their actions while performing a skill, experts, having proceduralized the requisite motor behaviors, do not require higherlevel processing to perform the skilled behavior. Habitualists further think that skilled behavior does not involve any higher-level cognition at the level of mastery.

While we accept many of these claims, and need not rehearse the evidence for them here, we resist the idea that level pluralism exclusively points to habitualism. Instead, we agree with other critics of habitualism that the increase in automaticity may provide experts with additional ways to engage in higher-level cognition during skilled behavior, which is best understood through the tools of intellectualism. To illustrate the point, we use the case of mind wandering during skilled behavior. While our argument is somewhat speculative, we use it in part to demonstrate our claim that higher-level cognition can be about content or control. Previous work by intellectualists has focused on the increase of an expert's control during skilled behavior, but we demonstrate here the presence of higher-level cognition in skilled behavior through the case of mind wandering, a form of spontaneous cognition. By doing so, we offer a case that we think should be accepted by habitualists and intellectualists alike.

Christoff, et al. (2016) define mind wandering as a class of spontaneously generated thoughts characterized by their relative instability and lack of attentional constraint. Because mind wandering typically occurs when attentional constraints are low, it is a promising candidate for a type of cognitive processing that may occur alongside automatic, skilled behaviors. Consider everyday tasks such as doing the dishes and driving a car - during these habitual tasks our minds frequently wander, musing on or imagining events other than those before us. This demonstrates that higher-level cognition can occur alongside skilled behavior. Supporting this idea, there is evidence 
that practice decreases the dependence on executive control and working memory, and that mind wandering tends to occur in just such cases (Weissman et al. 2002; Smallwood et al. 2003). This is why everyday tasks are highly susceptible to mind wandering episodes - as skill level increases, actions become increasingly proceduralized, and this proceduralization leads to a decrease in cognitive resources that need to be directed toward the task at hand. As a result of practice decreasing the cognitive load required to perform a given action, highly practiced individuals are more susceptible to mind wandering episodes. ${ }^{11}$

Note that we have so far described mind wandering in terms of cognitive processing that is unrelated to the primary, skilled task. Habitualists accept that automatic skilled behavior can occur alongside this form of higher-level cognition. In fact, they sometimes use higher-level cognition in a secondary task to prove the automaticity of the first (in so-called "dual-task" experiments). To see how this works, consider the differential effects of mind wandering on novices and experts. Because novices have not integrated actions into their procedural memory, mind wandering is likely to have a detrimental effect on their behavior. That is, because novices need to focus on their actions while performing, mind wandering may serve to distract them from the task at hand. However, experts, having integrated action sequences into their procedural memory such that their behavior may unfold automatically, are often able to successfully perform skilled behaviors while harboring thoughts with contents unrelated to the task at hand. Thus, the habitualist finds that different levels of skill correspond with different levels of task-related cognitive processing, but task-unrelated cognitive processing can increase as task-related cognitive processing decreases without cost to the skilled behavior. And so the overall level of higher-level cognition can remain the same for the novice and expert under habitualism, so long as higher-level cognition is task-related in the first case and task-unrelated in the second.

Contrary to habitualism, we think the presence of mind wandering in everyday tasks demonstrates room for task-related higher-level cognition in automatic skilled behavior, too. This is because mind wandering can contribute to the task at hand; the benefits of mind wandering - e.g. prospection and creativity (Smallwood and Schooler 2015) apply just as well to skilled behavior as to other behaviors. For example, mind wandering episodes can facilitate skilled behavior via the creativity of insight. Zedelius and Schooler (2015) found that increased rates of mind wandering were positively associated with a creative problem solving task when participants approached the problem with insight. However, this benefit disappeared when participants approached the problem with a more deliberate, analytic strategy. This suggests that the taskdemands and specific cognitive strategies employed during skilled behavior may modulate the usefulness of mind wandering during skilled behavior. For example, in tasks with a predefined end or goal, an analytic strategy or rigid set of automatic, rehearsed behaviors may result in successful skilled behavior; however, in tasks that are more open-ended and require creative solutions, allowing one's spontaneous cognitions to influence the unfolding behavior may be beneficial.

This becomes more clear if we recall the expert knitter from the introduction who spontaneously thinks about their friend's stripe preference during a mind wandering

\footnotetext{
${ }^{11}$ Of course, all individuals are susceptible to mind wandering episodes when not engaged in a task. Thanks to an anonymous reviewer for pointing this out.
} 
episode. "Ah-ha!" they say. After a series of spontaneous thoughts and a sudden insight, they begin knitting a hat with alternating stitch rows, rather than the planned single stitch hat. In this example, we see that spontaneously generated thoughts that may seem initially unrelated to one's task can be seamlessly integrated into the task at hand. In general, we might imagine the expert who desires to push the boundaries of their expertise and do something more creative than usual (Montero's kaizen). The expert may find that privileging internal trains of thought at certain times gives them novel ideas for how to proceed with the skilled behavior. So while it may be tempting to consider mind wandering as solely detrimental to skilled behavior (perhaps based on your experience operating a motor vehicle), we believe that in some cases, especially moments of insight, mind wandering can facilitate skilled behavior.

A further reason that mind wandering might be task related during skilled behavior is due to its prospective nature: it is often about future possible events. Baird et al. (2012), drawing on data that mind wandering has this prospective bias, explored the hypothesis that one potential function of mind wandering is to plan and anticipate future goals. The data suggest he is right: half of the mind wandering episodes reported were about the future and half of those were related to a subject's goals. We find it likely that prospectively-oriented mind wandering episodes could bolster an agent's capacity for situation-specific responding in skilled behavior. Baird himself suggests these prospective mind wandering episodes may help individuals navigate their daily lives, and we think the same is likely to be true for skilled behavior. Morsella et al. (2010)'s work on spontaneous thoughts about future tasks also supports this suggestion. When participants are told of an upcoming difficult task before the breathing exercise, they report more task-related spontaneous cognitions than when told of an upcoming simple task. Therefore, this suggests, in challenging situations that might benefit from forethought, prospective mind wandering episodes may serve a preparatory role for the skilled actor.

At this point, an intellectualist could respond that prospective mind wandering episodes are not relevant because they precede the skilled behavior in question. ${ }^{12}$ However, one can imagine a case in which someone initiates a skilled behavior, has a mind wandering episode (post-initiation) which contains an insight about how to confront an anticipated obstacle, and then adjusts their behavior accordingly in preparation for the event. Note that a mind wandering episode such as this is distinct from deliberate rehearsal before a performance: the former is spontaneous and occurs postinitiation and the latter is deliberate and occurs pre-initiation. So it appears that, at least in some circumstances, it is possible that mind wandering can serve a sort of "on the fly" rehearsal that may aid skilled behavior.

So we agree with the intellectualists that higher-level cognition plays an important role in skilled behavior, but, as we see it, both spontaneous and deliberate cognition can play this role. Recall again Montero's kaizen: experts are able to deliberately think about what they are doing in a manner that pushes the boundaries of their expertise. We want to suggest that spontaneous cognition can do the same. This is clear when considering the agent who, while directing attention away from the external task environment to their internal stream of consciousness, has an insight that leads to improvement in their skilled behavior. In further support of this, Baird (2012) found

$\overline{12}$ Thank you to an anonymous reviewer for suggesting this. 
that participants who reported more mind wandering episodes performed better at a divergent thinking task than those who reported less mind wandering. In a similar study, Preiss et al. (2016) found that mind wandering predicted creativity, but metacognition did not.

Taken together, the above studies suggest that mind wandering can count as taskrelated higher-level cognition and can aid in finding novel, creative solutions to problems that arise during skilled behavior. We might adjust the claim of level pluralism to the view that different levels of skill correspond with different degrees of required cognitive processing.

Even if task-related mind wandering can occur alongside skilled behavior, there is still an open question as to whether the contributions of mind wandering to skilled behavior count as part of skilled behavior. According to Bergamin, it is obvious that higher-level cognition can occur between moments of performing expert "micro-tasks"; however, what is in question is if there is such processing in the moment of performing these tasks, or only in the "gaps between" them. Bergamin describes the switch between skilled behavior and higher-level cognition as occurring very frequently: "we slip from moments of smooth-coping into reflective thought and back again at incredibly frequent intervals" (412). We find this carving up of skilled behavior at the line between higher-level cognition and automaticity to be somewhat arbitrary; we find it more natural to treat skilled behavior as continuous, and thus as including task-related mind wandering (see also Section 6).

Recall that Bergamin also discusses the "gaps above" skilled behavior; this idea aligns with our concept of task-unrelated higher-level cognition. The idea is that taskunrelated higher-level cognition need not count as part of skilled behavior. Bergamin cites the expert musicians in Høffding (2014) who describe the experience as like "having two tracks running" (65). In our view, we should count higher-level cognition as part of skilled behavior only if its content is task-relevant, such that it would be incorrect to describe the case as one of parallel processing, or "two tracks running." There may be moments of skilled behavior that do not include higher-level cognition of this sort, as Bergamin suggests, but we contend that there are likely to be other moments that do.

For all of the above reasons, level pluralism does not clearly support habitualism at the expense of intellectualism; for spontaneous cognition, it depends on how "normally" our mind wandering episodes are task-related in skilled behavior, which is a question we cannot currently answer. As it stands, we find both views to explain different aspects of level pluralism: habitualists are able to explain the reduction in required cognitive processing, but intellectualists are able to explain the continued benefit of higher-level cognition.

\section{Synchronic Pluralism}

Whereas level pluralism is the stance that the degree of cognitive processing in skilled behavior normally varies by the level of skill, synchronic pluralism is the stance that the degree of cognitive processing depends on the part or aspect of the behavior in question, combining insights from habitualism and intellectualism. Since these parts or aspects of skill co-occur at any given time through vertical layering, this is called 
"synchronic" pluralism. In contrast, diachronic pluralism, which we cover in the next section, is the stance that the degree of cognitive processing will vary over the time course of a skilled behavior (through horizontal layering).

In the last section we used the case of mind wandering to discuss level pluralism. In this section we will use the case of top-down attention to explain synchronic pluralism, using a framework provided by Papineau (2015). ${ }^{13}$ Top-down attention here refers to the process of mental selection that is based, at least in part, on prefrontal feedback, most commonly associated with voluntary, task-based attention (see, e.g., Jennings 2012). Both top-down attention and mind wandering will come up again in the discussion of diachronic pluralism, in the next section.

Papineau discusses a puzzle about the role of attention in skilled behavior: focus and concentration seem essential for skilled behavior, yet some have found attention to skill to degrade performance of that skill. Montero, for example, argues that experts "often do and should pay attention to or focus on what they are doing" (Montero 2016, 214). Beilock and Carr, on the other hand, find in a number of experiments that "although close attention and control may benefit novice performers in the initial stages of task learning, it will become counterproductive as practice builds a more and more automated performance repertoire" $(2001,715)$. Papineau solves this problem by separating attention to the "basic action" from attention to its "components" (Papineau 2015). ${ }^{14} \mathrm{~A}$ "basic action" is the action an agent intends to perform, whereas its "components" are lower level behaviors that make up the basic action. Papineau thinks that attention is required for the proper execution of basic actions (à la intellectualism), but that attention to its components degrades skilled performance (à la habitualism).

Papineau's account has close ties with level pluralism, since what counts as a basic action or component depends on one's level of skill. Yet, even given a specific level of skill there will be parts or aspects of one's behavior that differentially depend on cognitive processing, hence synchronic pluralism. Take the example of knitting introduced above. At the beginning of learning to knit a stitch, the basic actions include moves like "slide the point of the right needle through the first loop on the left needle from front to back (and from left to right) so that the two make an X" (Stoller 2003, 34). One needs to pay attention to each aspect of these instructions in order to succeed in forming this first part of the stitch. As one becomes familiar with the move, the basic action becomes "insert right needle," since the way that one inserts the right needle is habitual and automatic. Now if one were to pay attention to each aspect of inserting the right needle the move would seem unnatural and the stitch is likely to be less good than if attention had remained at the level of the basic action. The knitter thus normally uses higher-level cognition for the basic action, but not its components, and what the basic action is changes with practice. This is what we are calling synchronic pluralism, which makes use of vertical layering.

In terms of empirical evidence, there is ample support for Papineau's framework, and for a claim of synchronic pluralism. Yamaguchi et al. (2016), for example, describe how typists "chunk" the components of their basic actions:

\footnotetext{
${ }^{13}$ An anonymous reviewer points out that it may be possible to read Papineau as also arguing for a form of diachronic pluralism.

14 This is a move that Montero appears to endorse (Montero 2016, 15).
} 
"Chunking allows division of the labor involved in typewriting, such that the higher level control focuses on language processing (e.g., comprehending and composing sentences), whereas the lower level control focuses on keystrokes (e.g., translating letters into finger movements and moving the fingers to the key locations). Memory chunking interfaces the higher and lower level processes by associating one higher level unit with several lower level units, making parallel processing of keystrokes possible."

That is, the behavior of typing has different synchronic parts, some of which require more cognitive processing; one might type a specific sentence using a high level of cognitive processing but at the same time execute specific movements using a low level of cognitive processing. Imagine, for example, that one is about to type the second letter of the sentence "the quick brown fox jumps over the lazy dog": typing the second letter of the sentence requires a high level of cognitive processing, but typing the $h$ key requires a low level of cognitive processing.

One way synchronic pluralism may be explored empirically is through the difference between a "proximal" and "distal" focus of attention. For certain tasks that are extended in space, the basic task of a novice may be relatively proximal, whereas the basic task of an expert may be relatively distal, with the proximal now a component of the basic task for the expert. The challenge is setting the level of difficulty such that what the experimenter treats as proximal and distal tracks the change from novice to expert. McKay and Wulf (2012), for example, found that novice dart throwing performance improved when attending to the target (distal focus), rather than the flight of the dart (proximal focus). Yet, Kearney (2015) found that a distal focus in their study was better for novice golfers only when performing simple putting tasks, but that proximal focus was better for difficult golfing tasks. In another study, distal focus was found to be best for expert kayak sprinters in even the most challenging conditions (Banks 2015).

As with level pluralism, habitualism and intellectualism help to explain different elements of synchronic pluralism; habitualism explains the automatization of some aspects of skilled behavior and why it is harmful to attend to those aspects (i.e. the components of basic action, for Papineau), while intellectualism explains the role of higher-level cognition in other aspects of skilled behavior and why it is beneficial to attend to those aspects (i.e. the basic action). So far, our reasoning is unlikely to raise many eyebrows: both level pluralism and synchronic pluralism have been discussed in other papers, under different names. Less commonly mentioned is the possibility of diachronic pluralism. This is the idea that the level of cognitive processing in skilled behavior will vary over the time course of that behavior. We discuss diachronic pluralism in the next section, making the case that it challenges both habitualism and intellectualism.

\section{Diachronic Pluralism}

Diachronic pluralism intersects with synchronic pluralism, since the parts or aspects of skilled behavior that require a high level of cognitive processing in synchronic pluralism typically occur over longer spans of time-knitting a stitch takes longer than inserting a knitting needle. We can nonetheless distinguish synchronic from diachronic pluralism by noting that beginning a stitch by inserting a knitting needle takes the same 
amount of time as just inserting a knitting needle despite the former requiring more cognitive resources. Whereas synchronic pluralism is the idea that different parts or aspects of skilled behavior correspond with different degrees of cognitive processing, diachronic pluralism is the idea that different moments in the time course of skilled behavior correspond with different degrees of cognitive processing. That is, diachronic pluralism makes use of horizontal layering, rather than vertical layering, in a manner that will be explained below. In this section, we discuss diachronic pluralism through both attention and mind wandering.

As mentioned above, top-down attention and mind wandering are often described as opposed to one another, with mind wandering occurring just when attention has lapsed: "Mind-wandering, or those transient periods of time during which our attention momentarily drifts away from our on-going task and perceptual milieu, is fundamental to human neurocognitive function" (Kam et al. 2012; see also Gonçalves et al. 2018). ${ }^{15}$ Imagine that while knitting a stitch you find yourself momentarily thinking about an email you need to send when a clack of the needles brings your attention back to the stitch. We are not always aware of mind wandering, and it may be that mind wandering is more potent when we are not aware of it (Christoff et al. 2009). Yet, when we are aware of mind wandering it is often clear that our mind has shifted away from the task. In that situation, assuming this is not a case of divided attention, we are not using topdown attention to perform the skilled behavior (to knit the stitch).

Mind wandering is seen as normally periodic for healthy brain function: "Our data suggest that when trying to engage attention in a sustained manner, the mind will naturally ebb and flow in the depth of cognitive analysis it applies to events in the external environment" (Smallwood et al. 2008; see also Smith et al. 2018). Thus, even in the case that knitting requires attention, we are likely to have moments during knitting when our mind is elsewhere engaged, as in the example. In those cases, at those moments, we are not using higher-level cognition to perform the skilled behavior. ${ }^{16}$

The periodicity of attention itself is frequently asserted by researchers on the topic: "Sustained attention naturally fluctuates, with a periodicity in the theta $(4-8 \mathrm{~Hz})$ frequency range" (VanRullen 2013). The phenomenon is nicely described by Sutton, who discusses the skilled behavior of batting in cricket: "Attention in batting is pulsing rather than absent" (2007, 775). Support for the idea can be found, for example, in research on attentional blink. Attentional blink is a phenomenon in which a second target is missed if it appears within half a second of the first target. As Nieuwenstein, Potter, and Theeuwes argue, "the root cause of the blink lies in the difficulty of engaging attention twice within a short period of time for two temporally discrete target events" (2009). Skill training can reduce the impact of this effect, and perhaps even eliminate it for a specific time window, but this does not mean that attention altogether loses its periodicity in skilled behavior (see, e.g., Oei and Patterson 2015; Choi et al. 2012). Instead, we think it likely that the pacing of attention is adjusted as one learns a skill to best match the requirements of the task.

\footnotetext{
${ }^{15}$ Some distinguish the form of attention lost in mind wandering with other forms of attention that may be present in mind wandering, such as "internal" attention or "unguided" attention (Chun et al. 2011; Irving 2016). We set aside here the purposeful mind wandering that is central to these accounts in order to focus on the periodic mind wandering that occurs while performing a task.

${ }^{16} \mathrm{We}$ are assuming in this example that the mind-wandering is task-unrelated.
} 
This observed periodicity of mind wandering and top-down attention is enough to suspect diachronic pluralism, or the idea that the degree of cognitive processing in skilled behavior depends on moment by moment changes in the time course of that behavior. This is because the phenomenon seems just as likely to occur in skilled behavior as in non-skilled behavior. At least, we have no reason to suspect that the periodicity stops with the onset of skilled behavior. ${ }^{17}$ Further, mechanistic explanations of this periodicity suggest that it extends to skilled behavior. In one such explanation, the default mode network that is active in mind wandering is governed by slow-wave oscillations, which compete with and sometimes disrupt task-related activity, such as that maintained by top-down attention (Sonuga-Barke and Castellanos 2007). In another, non-exclusive explanation, top-down attention is governed by slow-wave oscillations, and the troughs of these oscillations allow for interruption by spontaneous neural activity, such as the spontaneous neural activity seen in mind wandering (see, e.g., Saleh et al. 2010; West et al. 2012; Jennings 2020). These mechanistic explanations would apply just as well to skilled and non-skilled behavior, suggesting that mind wandering and top-down attention are periodic in skilled behavior. While mind wandering may be seen as a form of higher-level cognition, when it is not task-related the task is not benefitting from that higher-level cognition. Thus, in such moments, higherlevel cognition can be said to be absent, or nearly absent, from the skilled behavior (see Table 1 below). Because such moments seem to occur with some regularity, we find that both the presence and absence of higher-level cognition are a normal part of skilled behavior. $^{18}$

Some might object that moments of mind wandering should not be included in an inventory of skilled behavior, in keeping with Bergamin's reasoning (see Section 4). That is, one might argue that skilled behavior pulses with the control of attention, rather than continuing as attention waxes and wanes. We have three reasons to set aside this possibility. First, it seems ad hoc, since the behavior appears to continue even while the mind wanders; the knitter continues to knit. We take it that the pacing of attention can be modified by context, such that there need be no loss to behavior even in these momentary dips in control. Second, mind wandering sometimes plays an important role in skilled behavior, such as by contributing to creative problem solving, so it seems mistaken to exempt it from counting as a part of skilled behavior; consider the mind wandering knitter who thinks about their friend and is thus inspired to knit a striped hat (see also Baird et al. 2012). Third, since attention and mind wandering are naturally periodic, we would have to extend the pulsing claim to all action, which seems absurd. That is, we would have to say that all actions start and stop with the natural waxing and waning of attention, which occurs multiple times a second. We argue, instead, that skilled behavior and other forms of action continue while attention and mind wandering wax and wane, supporting our claim of diachronic pluralism.

Finally, some might object that metacognitive processing will be necessary to govern top-down attention, mind wandering, or the relationship between them. If

\footnotetext{
${ }^{17}$ The characteristics of this periodicity may nonetheless be modified by skill. Thanks to an anonymous reviewer for making this point.

${ }^{18}$ Again, our definition of "normal" is "as a rule." While it should be obvious why we see attention as present or absent as a rule in skilled behavior, we cannot at this point specify the rule that makes mind wandering sometimes task-related and sometimes task-unrelated, but we suspect that both will be a regular part of skilled behavior.
} 
Table 1 The supposed occurrence of attention and mind wandering for different cases of skilled behavior. The novice does not yet have automatized components, whereas the master has automatized all possible basic actions. The expert is in between, with some automatized components but also a basic action that is not yet automatized. Thus, attention is beneficial for the basic action but harmful for its components, and nonetheless periodic. Mind wandering is likewise periodic but beneficial or harmful depending on its content. Mind wandering is harmful for the novice because they cannot rely on automaticity, yet it is nonetheless periodic. Given the rarity of true novices and masters in skilled behavior, most instances of skilled behavior will be ones in which attention, mind wandering, and automaticity are all present as a rule

\begin{tabular}{lll}
\hline & Top-down attention & Mind wandering \\
\hline Novice, basic action & Periodic, beneficial & Periodic, harmful \\
Expert, components & Rare, harmful & Periodic, task-related/beneficial or task-unrelated/harmful \\
Expert, basic action & Periodic, beneficial & \\
Master, basic action & Rare, harmful & Rare, harmful \\
\hline
\end{tabular}

metacognitive processing is required for skilled behavior then cognitive processing would be high even in the case of momentary lapses of attention. Bermúdez argues something like this in a recent paper: "If you are acting skillfully, then you are reflecting, because performing a skilled action requires that throughout performance your attention is structured by a higher-order, performance-related intention" (2017). Similarly, Smallwood and Schooler (2015) argue that mind wandering must be monitored and controlled by a metacognitive state since mind wandering increases when task demands are low and decreases when task demands are high.

Against this, we do not think it is necessary to suppose metacognitive support for skilled behavior. Recall that Yamaguchi, et al. claim memory is the source of coordination between the parts or aspects of skilled behavior: "Memory chunking interfaces the higher and lower level processes by associating one higher level unit with several lower level units, making parallel processing of keystrokes possible." This removes the need for metacognitive processing to coordinate vertical layering, since the direct links of association are enough to coordinate the components of basic actions.

We think that horizontal layering is likewise achieved through lower-level mechanisms. The orthogonality of the so-called "task-positive" and "default-mode" networks in the brain, which roughly corresponds to the orthogonality between top-down attention and mind wandering, is such a basic feature of day-to-day cognition that its reduction is implicated in psychosis (Carhart-Harris et al. 2013). We think it unlikely that such a basic feature of day-to-day cognition is continuously maintained by a metacognitive state. At least, we see no reason to presuppose the existence of a higher-order process that maintains the relationship between attention and mind wandering at this stage (see also Fox et al. 2005).

The fact that mind wandering is linked to task demands, as noted by Smallwood and Schooler, might be explained by other factors. One possible factor is the real estate requirements of difficult tasks. That is, a difficult task likely uses more areas (or just more) of the brain than an easy task. Thus, mind wandering is more likely to happen during an easy task, since more of the brain is available to spontaneous cognition. Importantly, this explanation does not require a metacognitive state that monitors the demands of the task. 
In our view, the periodic nature of attention and mind wandering indicates the existence of diachronic pluralism, such that higher-level cognitive processing will normally ebb and flow throughout one's skilled behavior. This view puts pressure on habitualism and intellectualism: it holds that at most levels of skill there will be periodic higher-level cognition, putting pressure on the habitualist, and it holds that there will be periodic absences of higher-level cognition for skilled behavior, which the intellectualist denies. In the next section we review a special case that puts even more pressure on these accounts, since it more clearly demonstrates the features of diachronic pluralism: strategic automaticity.

\section{Strategic Automaticity}

A special case of diachronic pluralism is that of "strategic automaticity," as described in Jennings (2020). The term is used here to describe a situation in which the agent intentionally and strategically embarks on a sequence of automatic behavior. That is, in strategic automaticity the agent may use higher-level cognition to initiate the behavior, but not to perform it. This case is only possible for highly familiar behaviors in highly familiar contexts. Thus, this is a case of skilled behavior that requires higher-level cognition at one point in the time course of that behavior, but not in another.

Strategic automaticity is normally mentioned in terms of what are called "implementation intentions" or "if-then planned behavior," in which one intends to perform a certain behavior if a certain context arises. For example, while knitting the striped hat one might intend to "purl" a row once one has finished knitting a row. MartinyHuenger et al. (2015) make the case that such behaviors can be automatically initiated, since such initiation is efficient, effortless, and unconscious: "We will argue that humans can willfully exert automatic action control by an anticipatory process of consciously linking a goal-directed response to an anticipated situation (i.e., if-then planning)." In the example above, by setting the intention in advance to do a purl stitch once one reaches the end of a row, the behaviors required to do a purl stitch can be initiated automatically once the knitter perceives they have reached the end of the row.

Related phenomena are described in philosophical analyses of skill. Sutton (2007), for example, discusses the role of "instructional nudges" in cricket: "a complex bodily pattern or set of possible movements can be compressed into and partly cued by a phrase or memory or ingrained image, bringing the player back to, rather than away from, the welllearned habits." The idea is that certain phrases or cues are learned by athletes so that later they can use the phrases or cues to activate certain habitual patterns of behavior. Describing the same phenomenon, Buskell (2015) puts forward the case of "discursive cueing":

"Such cues act as context-sensitive guides, orienting the performer to salient aspects of the environment and of an on-going skilled performance. The important thing to note here is that discursive cuing straddles the automatic/nonautomatic divide. The actual triggering of the discursive cue can be automatic...But such routines function by being conspicuous: the discursive cue causes a focusing of attention because one notices or utters a phrase here, at this time."

Both Sutton and Buskell see this case as one of only partial automaticity. For Sutton, the habitual behavior is intentionally activated. For Buskell, it is automatically activated 
but in a way that directs attention. Our own example of strategic automaticity does without these elements, which we find to be consistent with the scientific literature on this case.

First, we do not find it necessary to invoke attention to explain the fact that habitual behavior is contextual. Recall that habitual behavior is thought to be driven by direct links between stimulus and response, or "S-R" associations. It has been demonstrated that S-R associations can be filtered through context, but this "does not depend on attention being directed to the context" (Bouton et al. 2014). Instead, "the context might be part of the stimulus that supports instrumental S-R habits" (Thrailkill and Bouton 2015). It is for this reason that we agree with Fridland (2017) that automaticity is distinct from reflex while disagreeing with her that this feature of automaticity requires attention.

Second, we see a role for the automatic initiation of habitual action sequences. Beyond the behavioral evidence, Martiny-Huenger et al. note that the initiation of behaviors caused by implementation intentions is associated with medial, rather than lateral prefrontal cortex: "Gilbert and colleagues found heightened activity in the lateral BA 10 for goal-driven behavior but heightened activity in the medial BA 10 for implementation intention-driven behavior" (2015). In their view, this supports their contention that implementation intentions can lead to the automatic initiation of behavior: "this evidence from cognitive neuroscience fits with the behavioral evidence and the assumptions underlying implementation intentions that if-then planning delegates a person's action control to situational cues" (Martiny-Huenger et al. 2015). Given the behavioral and neural evidence they provide, we see no reason to assume that the initiation of behaviors by implementation intentions would need to be intentional. (But note that this point is not a necessary part of our argument that strategic automaticity is possible, since as long as it is possible to strategically initiate an automatic action sequence then we have established diachronic pluralism, even if the initiation itself is not automatic.)

While Martiny-Huenger et al. are careful to distinguish implementation intentions from habitual behaviors, the combination might lead one to argue for skilled behavior in the total absence of higher-level cognition. In this version of strategic automaticity, the skilled behavior is not only automatically initiated, but automatically executed. This is because habitual behaviors are governed by subcortical mechanisms, such as by the striatum: "Multiple lines of evidence have supported the idea that the basal ganglia, specifically the dorsal striatum, mediate the acquisition and expression of S-R habits" (Knowlton and Patterson 2016). As mentioned above, goal-directed behaviors are standardly distinguished from habitual behaviors, the latter of which are stimulus-response pairings mediated by context (e.g. Thrailkill and Bouton 2015). In contrast, goal-directed behaviors depend on outcome evaluation-an instance of higher-level cognition (e.g. Gremel and Costa 2013). While there is some evidence that medial prefrontal cortex also plays a role in habitual behavior (e.g. Smith et al. 2012), the area of the medial prefrontal cortex that is associated with habitual behavior is distinct from that associated with goaldirected behavior (Killcross and Coutureau 2003).

Against this idea that strategic automaticity is a case for skilled behavior in the total absence of higher-level cognition, note that the implementation intentions are preceded by earlier intentions that depend on higher-level cognition. That is, even in this extreme case higher-level cognition is present to some 
degree, albeit at an earlier moment in time. This is what makes the automaticity strategic. $^{19}$

Strategic automaticity is an ideal case of diachronic pluralism because it explicitly combines the features of skilled behavior at issue here. It also accords well with reports from experts. Recall from the introduction that experts often describe what they do as "automatic." Yet, in all of the cases mentioned, the automaticity is described as strategic and intentional. One reason that experts may sometimes favor strategic automaticity is to avoid choking under pressure, mentioned above: performing a familiar behavior automatically helps to prevent interruption of that behavior by other cognitive processing. Chaffin and Logan (2006) describe this issue for classicallytrained musicians: "For memory to work reliably under the pressures of the concert stage, the performance must be practiced until it is thoroughly automatic." But even if the skill is automatically executed, it depends on an earlier moment of non-automatic cognitive processing, supporting the claim of diachronic pluralism.

Strategic automaticity is facilitated by chunking, described above. One way of thinking about chunking is that the cognitive processing for these behaviors is simply done in advance, at the beginning of the action sequence (e.g. Dezfouli and Balleine 2013). This is consistent with the finding that chunked sequences differ from nonchunked sequences in having a time lag before the first behavior in the sequence (e.g. Santos and Badre 1994). In this account, it would be appropriate to include the full time window, from planning to execution, in an analysis of whether or not the behavior requires higher-level cognition, since the cognitive processing has apparently been redistributed over that window.

Against this account, not only is the standardly observed time lag for chunked sequences shorter than one would expect on this account, some have found evidence that the time lag may not occur at all in those who are highly skilled. As described in Thompson et al. (2017):

"Athletes with medals in national or international competition showed no relationship between premotor time...and sequence complexity, while sub-elite athletes with a comparable number of years of experience were slightly faster preparing for a single kick than for kick sequences of length 3 or 6 (Chen et al., 2015). Our results appear to be another example of weakening relationships between response time and Action-Count."

Thus, it doesn't appear to be the case that the cognitive processing is simply redistributed over a longer time period, but that cognitive processing requirements are reduced for at least some parts of the time window. This fits the account of increasing automaticity described in earlier sections. Further, the familiarity that allows for chunking comes along with other changes in skill execution that put pressure on the redistribution account. These changes, which we briefly reviewed above, encourage an interpretation of strategic automaticity in which higher-level cognition is not required to execute the skilled behavior, even if it is required to initiate it.

Thus, strategic automaticity challenges the intellectualist idea that higher-level cognition is always present in skilled behavior to some degree. But it also puts pressure on the habitualist idea that peak performance is always automatic, absent of higher-

\footnotetext{
${ }^{19}$ Yet, as an anonymous reviewer notes, this may be much earlier than the implementation of the behaviors. It is our current view that even in the case of a long temporal gap between the planning and the initiation of these behaviors, it makes sense to include the planning phase as part of the behavior in question.
} 
level cognition. Instead, strategic automaticity is a case in which peak performance is at some point initiated by higher-level cognition but is ultimately executed automatically: a form of diachronic pluralism.

\section{Conclusion}

We have considered three varieties of pluralism with respect to skilled behavior: level pluralism, synchronic pluralism, and diachronic pluralism. All three are compatible with the empirical evidence on skill acquisition and skilled behavior. In the case of level pluralism, one becomes less reliant on higher-level cognition for skilled behavior as one gains mastery over that skill. Yet, evidence on mind wandering suggests that forms of higher-level cognition may nonetheless occur alongside skilled behavior, sometimes even contributing to the skilled behavior. In the case of synchronic pluralism, some aspects of a skill may unfold automatically whereas other aspects may require higher-level cognition. We used evidence on attention, and especially the distinction between proximal and distal focus of attention, to illustrate this idea. Finally, in the case of diachronic pluralism, we demonstrated that higher-level cognition naturally waxes and wanes, leaving moments in the time course of skilled behavior in which one is performing automatically even for demanding tasks. We combined evidence on attention and mind wandering to make this point.

We find that these cases challenge intellectualism and habitualism, taken alone. We suggest in their place a pluralist account, in which automaticity and higher-level cognition are woven together to facilitate skilled behavior. Some moments of skilled behavior may be fully automatic, and absent of higher-level cognition, whereas other moments of skilled behavior demand significant cognitive resources. Recall Montero's appeal to inconsistencies in first-person reports on skilled behavior: we think this is further evidence for pluralism. Experts sometimes claim to be performing automatically, but even in that case we find the strategic nature of their automaticity to require some distance from habitualism.

Acknowledgements Thanks to the Philosophy Lab at UC Merced, the anonymous reviewers, Aunt Jill for being a knitting inspiration, and participants at the Central Valley Philosophical Association, Southern Society for Philosophy and Psychology, and the Undergraduate Cognitive Science Conference for helpful guidance and feedback on the paper.

Open Access This article is licensed under a Creative Commons Attribution 4.0 International License, which permits use, sharing, adaptation, distribution and reproduction in any medium or format, as long as you give appropriate credit to the original author(s) and the source, provide a link to the Creative Commons licence, and indicate if changes were made. The images or other third party material in this article are included in the article's Creative Commons licence, unless indicated otherwise in a credit line to the material. If material is not included in the article's Creative Commons licence and your intended use is not permitted by statutory regulation or exceeds the permitted use, you will need to obtain permission directly from the copyright holder. To view a copy of this licence, visit http://creativecommons.org/licenses/by/4.0/.

\section{References}

Anderson, J.R. 1982. Acquisition of cognitive skill. Psychological Review 89 (4): 369-406.

Anderson, R., S.J. Hanrahan, and C.J. Mallett. 2014. Investigating the optimal psychological state for peak performance in Australian elite athletes. Journal of Applied Sport Psychology 26 (3): 318-333. 
Automaticity. Oxford Reference. Retrieved 16 Apr. 2020, from https://www.oxfordreference.com/view/10. 1093/oi/authority.20110803095436124.

Baird, B., J. Smallwood, M.D. Mrazek, J.W. Kam, M.S. Franklin, and J.W. Schooler. 2012. Inspired by distraction: Mind wandering facilitates creative incubation. Psychological Science 23 (10): 1117-1122.

Balleine, B.W., and A. Dezfouli. 2019. Hierarchical action control: Adaptive collaboration between actions and habits. Frontiers in Psychology 10: 2735.

Banks, S. D. (2015). Distal and proximal attentional focus effects on the performance of closed and open continuous motor skills (unpublished doctoral dissertation). University of Edinburgh.

Beilock, S.L., and T.H. Carr. 2001. On the fragility of skilled performance: What governs choking under pressure? Journal of Experimental Psychology: General 130 (4): 701.

Beilock, S.L., and R. Gray. 2007. Why do athletes choke under pressure? In Handbook of sport psychology, ed. G. Tenenbaum and R.C. Eklund, 425-444. Inc: John Wiley \& Sons.

Beilock, S.L., T.H. Carr, C. MacMahon, and J.L. Starkes. 2002. When paying attention becomes counterproductive: Impact of divided versus skill-focused attention on novice and experienced performance of sensorimotor skills. Journal of Experimental Psychology. Applied 8 (1): 6.

Bergamin, J.A. 2017. Being-in-the-flow: Expert coping as beyond both thought and automaticity. Phenomenology and the Cognitive Sciences 16 (3): 403-424.

Bermúdez, J.P. 2017. Do we reflect while performing skillful actions? Automaticity, control, and the perils of distraction. Philosophical Psychology 30 (7): 896-924.

Bouton, M.E., T.P. Todd, and S.P. León. 2014. Contextual control of discriminated operant behavior. Journal of Experimental Psychology Animal learning and Cognition 40 (1): 92-105. https://doi.org/10.1037/ xan0000002.

Buskell, A. 2015. How to be skilful: Opportunistic robustness and normative sensitivity. Synthese 192 (5): 1445-1466.

Caiani, S.Z., and G. Ferretti. 2017. Semantic and pragmatic integration in vision for action. Consciousness and Cognition 48: 40-54.

Carhart-Harris, R.L., R. Leech, D. Erritzoe, T.M. Williams, J.M. Stone, J. Evans, D.J. Sharp, A. Feilding, R.G. Wise, and D.J. Nutt. 2013. Functional connectivity measures after psilocybin inform a novel hypothesis of early psychosis. Schizophrenia Bulletin 39 (6): 1343-1351.

Chaffin, R., and T. Logan. 2006. Practicing perfection: How concert soloists prepare for performance. Advances in Cognitive Psychology 2 (2-3): 113-130.

Chang, Y. 2014. Reorganization and plastic changes of the human brain associated with skill learning and expertise. Frontiers in Human Neuroscience 8: 35.

Chen, C.Y., I. Jing Dai, F. Chen, K.M. Chou, and C.K. Chang. 2015. Reliability and validity of a dual-task test for skill proficiency in roundhouse kicks in elite taekwondo athletes. Open access Journal of Sports Medicine 6: 181.

Choi, H., L.H. Chang, K. Shibata, Y. Sasaki, and T. Watanabe. 2012. Resetting capacity limitations revealed by long-lasting elimination of attentional blink through training. Proceedings of the National Academy of Sciences 109 (30): 12242-12247.

Christensen, W., J. Sutton, and D.J. McIlwain. 2016. Cognition in skilled action: Meshed control and the varieties of skill experience. Mind \& Language 31 (1): 37-66.

Christoff, K., A.M. Gordon, J. Smallwood, R. Smith, and J.W. Schooler. 2009. Experience sampling during fMRI reveals default network and executive system contributions to mind wandering. Proceedings of the National Academy of Sciences 106 (21): 8719-8724.

Christoff, K., Z.C. Irving, K.C. Fox, R.N. Spreng, and J.R. Andrews-Hanna. 2016. Mind-wandering as spontaneous thought: A dynamic framework. Nature Reviews Neuroscience 17 (11): 718-731.

Chun, M.M., J.D. Golomb, and N.B. Turk-Browne. 2011. A taxonomy of external and internal attention. Annual Review of Psychology 62: 73-101.

Csikszentmihalyi, M. 1997. Flow and the psychology of discovery and invention. New York: Harper Perennial.

DeCaro, M.S., R.D. Thomas, N.B. Albert, and S.L. Beilock. 2011. Choking under pressure: Multiple routes to skill failure. Journal of Experimental Psychology: General 140 (3): 390-406.

Dezfouli, A., and B.W. Balleine. 2013. Actions, action sequences and habits: Evidence that goal-directed and habitual action control are hierarchically organized. PLoS Computational Biology 9 (12): e1003364.

Dreyfus, H.L. 2007. The return of the myth of the mental. Inquiry 50 (4): 352-365.

Dreyfus, S. E., \& Dreyfus, H. L. (1980). A five-stage model of the mental activities involved in directed skill acquisition (no. ORC-80-2). California Univ Berkeley Operations Research Center. 
Fox, M.D., A.Z. Snyder, J.L. Vincent, M. Corbetta, D.C. Van Essen, and M.E. Raichle. 2005. The human brain is intrinsically organized into dynamic, anticorrelated functional networks. Proceedings of the National Academy of Sciences 102 (27): 9673-9678.

Fridland, E. 2017. Skill and motor control: Intelligence all the way down. Philosophical Studies 174 (6): 1539-1560.

Gibson, J.J. 2014. The ecological approach to visual perception: Classic edition. Psychology Press.

Gonçalves, Ó.F., G. Rêgo, T. Conde, J. Leite, S. Carvalho, O.M. Lapenta, and P.S. Boggio. 2018. Mind wandering and task-focused attention: ERP correlates. Scientific Reports 8 (1): 1-14.

Gremel, C.M., and R.M. Costa. 2013. Orbitofrontal and striatal circuits dynamically encode the shift between goal-directed and habitual actions. Nature Communications 4: 2264.

Høffding, S. 2014. What is skilled coping?: Experts on expertise. Journal of Consciousness Studies 21 (9-10): 49-73.

Irving, Z.C. 2016. Mind-wandering is unguided attention: Accounting for the "purposeful" wanderer. Philosophical Studies 173 (2): 547-571.

Jennings, C.D. 2012. The subject of attention. Synthese 189 (3): 535-554.

Jennings, Carolyn Dicey. 2015. Consciousness without attention. Journal of the American Philosophical Association 1 (2): 276-295.

Jennings, C.D. 2020. The attending mind. Cambridge University Press.

Kam, J.W., E. Dao, P. Blinn, O.E. Krigolson, L.A. Boyd, and T.C. Handy. 2012. Mind wandering and motor control: Off-task thinking disrupts the online adjustment of behavior. Frontiers in Human Neuroscience 6: 329.

Kearney, P.E. 2015. A distal focus of attention leads to superior performance on a golf putting task. International Journal of Sport and Exercise Psychology 13 (4): 371-381.

Killcross, S., and E. Coutureau. 2003. Coordination of actions and habits in the medial prefrontal cortex of rats. Cerebral Cortex 13 (4): 400-408.

Killingsworth, M.A., and D.T. Gilbert. 2010. A wandering mind is an unhappy mind. Science 330 (6006): 932-932.

Knowlton, B.J., and T.K. Patterson. 2016. Habit Formation and the Striatum. In Habit formation and the striatum, In Behavioral neuroscience of learning and memory (pp. 275-295). Cham: Springer.

Krakauer, J.W. 2019. The intelligent reflex. Philosophical Psychology 32 (5): 822-830.

Levy, Neil. 2017. Embodied savoir-faire: Knowledge-how requires motor representations. Synthese 194 (2): 511-530.

Martiny-Huenger, T., S.E. Martiny, and P.M. Gollwitzer. 2015. Action control by if-then planning. The Sense of Agency: 63-94.

McKay, B., and G. Wulf. 2012. A distal external focus enhances novice dart throwing performance. International Journal of Sport and Exercise Psychology 10 (2): 149-156.

Mediocre AB. (2014). Smash Hit [mobile phone game].

Miller, E.K., and J.D. Wallis. 2009. Executive function and higher-order cognition: Definition and neural substrates. Encyclopedia of Neuroscience 4 (99-104).

Montero, B. 2010. Does bodily awareness interfere with highly skilled movement? Inquiry 53 (2): 105-122.

Montero, B.G. 2016. Thought in action: Expertise and the conscious mind. Oxford University Press.

Morsella, E., A. Ben-Zeev, M. Lanska, and J.A. Bargh. 2010. The spontaneous thoughts of the night: How future tasks breed intrusive cognitions. Social Cognition 28 (5): 641-650.

Mylopoulos, M., and E. Pacherie. 2017. Intentions and motor representations: The interface challenge. Review of Philosophy and Psychology 8 (2): 317-336.

Nieuwenstein, M.R., M.C. Potter, and J. Theeuwes. 2009. Unmasking the attentional blink. Journal of Experimental Psychology: Human Perception and Performance 35 (1): 159-169.

Oei, A.C., and M.D. Patterson. 2015. Enhancing perceptual and attentional skills requires common demands between the action video games and transfer tasks. Frontiers in Psychology 6: 113.

Papineau, D. 2015. Choking and the yips. Phenomenology and the Cognitive Sciences 14 (2): 295-308.

Preiss, D.D., D. Cosmelli, V. Grau, and D. Ortiz. 2016. Examining the influence of mind wandering and metacognition on creativity in university and vocational students. Learning and Individual Differences 51: 417-426.

Saleh, M., J. Reimer, R. Penn, C.L. Ojakangas, and N.G. Hatsopoulos. 2010. Fast and slow oscillations in human primary motor cortex predict oncoming behaviorally relevant cues. Neuron 65 (4): 461-471.

Santos, P. J., \& Badre, A. N. (1994). Automatic chunk detection in human-computer interaction. In Proceedings of the Workshop on Advanced Visual Interfaces (pp. 69-77). ACM.

Schneider, W., and R.M. Shiffrin. 1977. Controlled and automatic human information processing: I. detection, search, and attention. Psychological review 84 (1): 1.

Smallwood, J., and J.W. Schooler. 2015. The science of mind wandering: Empirically navigating the stream of consciousness. Annual Review of Psychology 66: 487-518. 
Smallwood, J.M., S.F. Baracaia, M. Lowe, and M. Obonsawin. 2003. Task unrelated thought whilst encoding information. Consciousness and Cognition 12 (3): 452-484.

Smallwood, J., E. Beach, J.W. Schooler, and T.C. Handy. 2008. Going AWOL in the brain: Mind wandering reduces cortical analysis of external events. Journal of Cognitive Neuroscience 20 (3): 458-469.

Smith, K.S., A. Virkud, K. Deisseroth, and A.M. Graybiel. 2012. Reversible online control of habitual behavior by optogenetic perturbation of medial prefrontal cortex. Proceedings of the National Academy of Sciences 109 (46): 18932-18937.

Smith, G.K., C. Mills, A. Paxton, and K. Christoff. 2018. Mind-wandering rates fluctuate across the day: Evidence from an experience-sampling study. Cognitive research: principles and implications 3 (1): 54.

Sonuga-Barke, E.J., and F.X. Castellanos. 2007. Spontaneous attentional fluctuations in impaired states and pathological conditions: A neurobiological hypothesis. Neuroscience \& Biobehavioral Reviews 31 (7): 977-986.

Stanley, J., and J.W. Krakauer. 2013. Motor skill depends on knowledge of facts. Frontiers in Human Neuroscience 7: 503.

Stoller, D. 2003. Stitch ' $n$ bitch: The Knitter's handbook. Workman Publishing.

Sutton, J. 2007. Batting, habit and memory: The embodied mind and the nature of skill. Sport in Society 10 (5): 763-786.

Taylor, N., and J. Elam. 2018. 'People are robots, too': Expert gaming as autoplay. Journal of Gaming \& Virtual Worlds 10 (3): 243-260.

Thrailkill, E.A., and M.E. Bouton. 2015. Contextual control of instrumental actions and habits. Journal of Experimental Psychology: Animal Learning and Cognition 41 (1): 69.

Thompson, J.J., C.M. McColeman, E.R. Stepanova, and M.R. Blair. 2017. Using video game telemetry data to research motor chunking, action latencies, and complex cognitive-motor skill learning. Topics in Cognitive Science 9 (2): 467-484.

Thrailkill, E.A., and M.E. Bouton. 2015. Contextual control of instrumental actions and habits. Journal of Experimental Psychology: Animal Learning and Cognition 41 (1): 69-80.

VanRullen, R. 2013. Visual attention: A rhythmic process? Current Biology 23 (24): R1110-R1112.

Weissman, D.H., M.G. Woldorff, C.J. Hazlett, and G.R. Mangun. 2002. Effects of practice on executive control investigated with fMRI. Cognitive Brain Research 15 (1): 47-60.

West, R., K. Bailey, B.N. Tiernan, W. Boonsuk, and S. Gilbert. 2012. The temporal dynamics of medial and lateral frontal neural activity related to proactive cognitive control. Neuropsychologia 50 (14): 3450-3460.

Wu, W. 2011. Attention as selection for action, 97. Attention: Philosophical and Psychological Essays.

Yamaguchi, M., J. Randle, and G.D. Logan. 2016. Pushing typists back on the learning curve: Memory chunking in the hierarchical control of skilled typewriting. Journal of Experimental Psychology: Learning, Memory, and Cognition 42 (12): 1919.

Yamashita, Y., and J. Tani. 2008. Emergence of functional hierarchy in a multiple timescale neural network model: A humanoid robot experiment. PLoS Computational Biology 4 (11): e1000220.

Zedelius, C.M., and J.W. Schooler. 2015. Mind wandering "Ahas" versus mindful reasoning: Alternative routes to creative solutions. Frontiers in Psychology 6: 834.

Publisher's Note Springer Nature remains neutral with regard to jurisdictional claims in published maps and institutional affiliations. 